\title{
Multi-scale Investigation of the Hydrogen-Assisted Failure of X65 Pipeline Steel
}

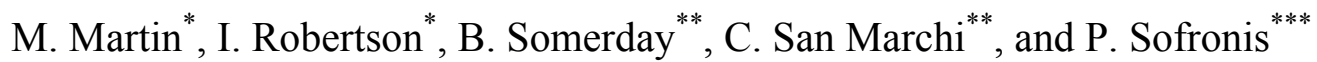

*Dept. of Materials Science and Engineering, U of Illinois - Urbana-Champaign, 1304 W Green St, Urbana, IL 61801

** Sandia National Laboratories, P.O. Box 969, Livermore, CA, 94550-0969

${ }^{* * *}$ Dept. of Mechanical Science and Engineering, U of Illinois - Urbana-Champaign, $1206 \mathrm{~W}$ Green St, Urbana IL 61801

Hydrogen is known to degrade the mechanical properties of steels and in some systems to cause a change in the transgranular fracture mode from ductile to either quasi-cleavage - facets not parallel to cleavage planes - or cleavage; a transition from a transgranular to an intergranular failure mode occurs in other systems $[1,2]$. Understanding how hydrogen-induced changes in either the lattice cohesive strength or in the properties of dislocations relate to the observed failure modes is needed to inform the development of physically-based continuum models that are capable of assessing the safety of metals used in hydrogen environments. For example, it was proposed to use the natural gas pipeline distribution system to transport hydrogen from remote production facilities to end-user stations. However, the susceptibility to hydrogen embrittlement of the wide range of steels used in constructing the natural gas pipeline system is unknown.

Compact tension specimens of API Grade X65 HIC pipeline steel were tested at Sandia National Laboratory (Livermore) in $20 \mathrm{MPa}$ hydrogen gas. An example of the resultant fracture surface is shown in Figure 1a. Some regions exhibit facet-like features and others are flat with features that resemble river patterns - the latter fracture surface, shown at higher magnification in Figure $1 \mathrm{~b}$, is commonly characterized as quasi-cleavage [3]. Such surfaces are not unique to this system as similar features have been observed on fracture surfaces of X70/X80 pipeline steels tested under this condition. To understand the formation of the quasi-cleavage surfaces, focused ion beam machining was used to extract samples from specific locations to enable determination of the microstructure immediately beneath the fracture surface. In this way, a correlation between fracture surface and microstructural features, such as secondary phases, dislocation structures, sub-grain boundaries can be made. In addition, the same area of sample was viewed over a wide angular range with images captured every few degrees of tilt and these were used to create a topographical map.

The series of images in the tilt sequence and the surface topography map reveal the ridges on the quasi-cleavage fracture surface protrude from the surface and show that saw-tooth like features decorate the ridge peaks. These features are evident in the fractograph presented in Figure 2. They appear thin and are reminiscent of saw-tooth features produced during the final stages of separation in an electron transparent section bounded by electron opaque regions, Figure 3 . In the latter case, the saw-teeth edges parallel slip or twinning planes and are attributed to the crack propagating along the slip band or the twin plane. This interpretation of the features on fracture surface suggests they are formed as a consequence of the elongation and growth of parallel channels with the ridges forming the walls between the channels. The nature of these channels 
along with the initiation sites remain to be identified. Preliminary analysis of the microstructure immediately beneath the fracture surface reveals no distinguishing features in the vicinity of the ridges or the flat surfaces. Identification of the initiation site and mechanism as well as the growth processes is needed to resolve why quasi-cleavage surfaces are produced on a hydrogeninduced fracture surface.

The authors acknowledge financial support from the Department of Energy, EERE, and the use of the facilities in the Center of Microanalysis of Materials at the University of Illinois.
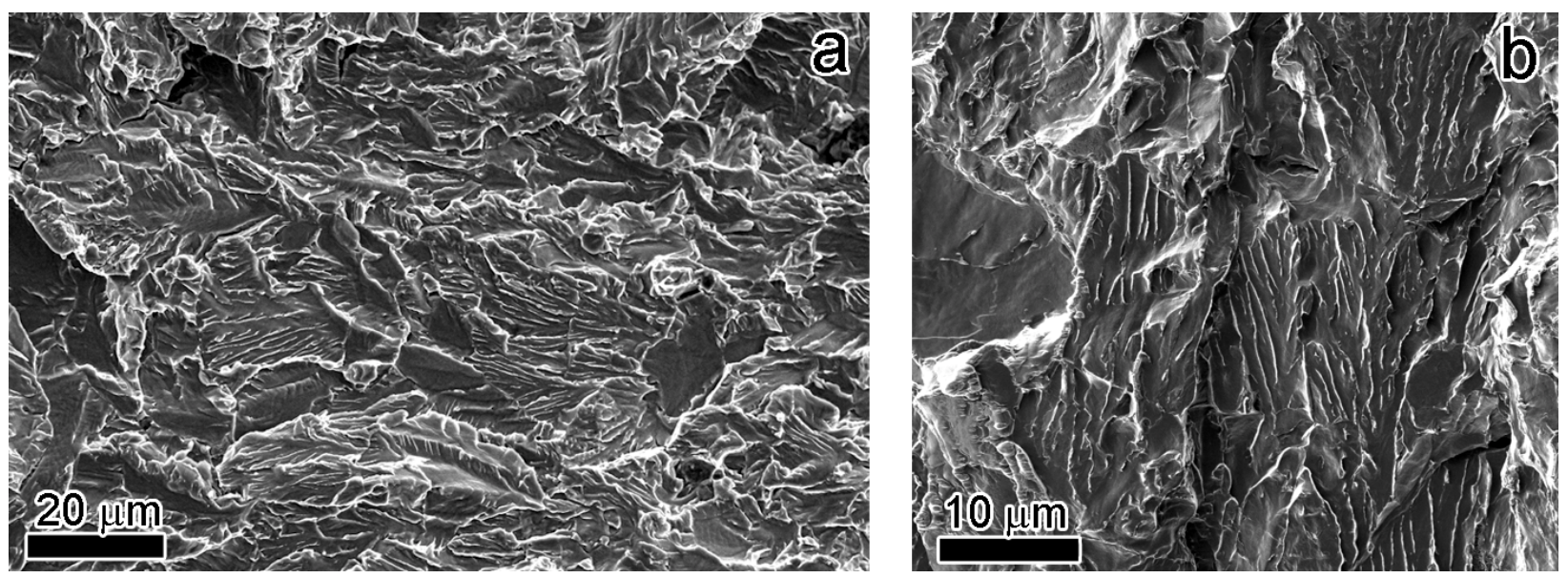

FIG 1a. SEM wide view showing features on fracture surface. b. Higher magnification SEM of feature of interest.

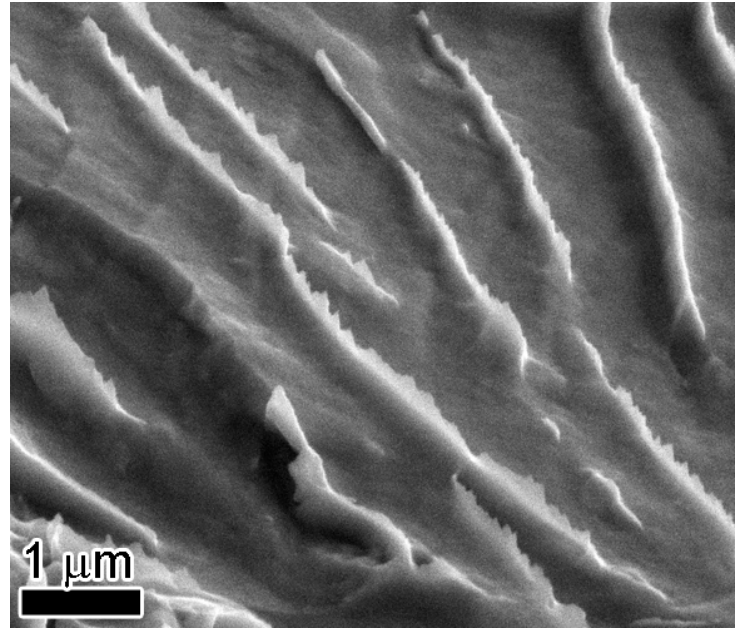

FIG 2. Fractograph showing the ridges with the saw-teeth on the ridge peaks.

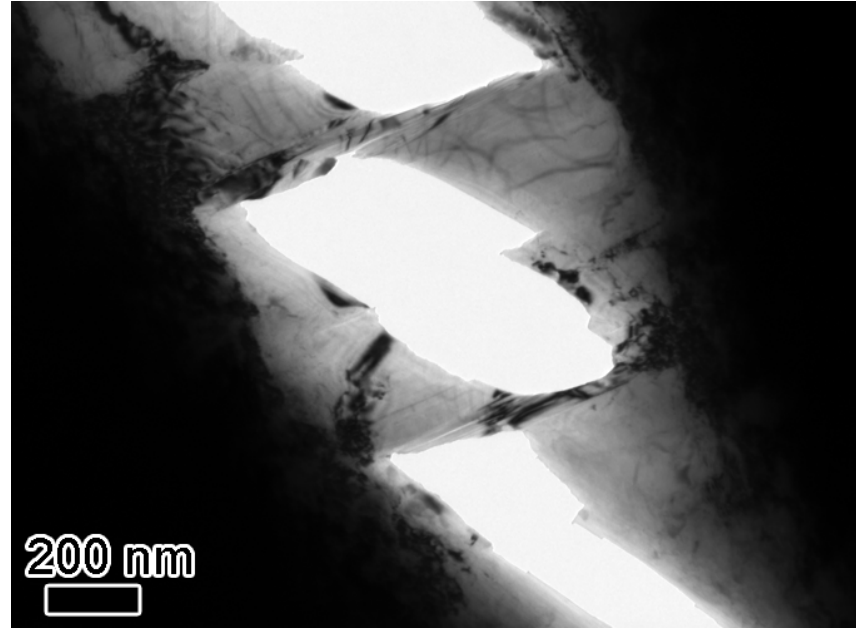

FIG 3. TEM image of $i$-situ fractured $\mathrm{Cu}-3 \% \mathrm{Co}$ sample showing saw-teeth due to tearing. Image courtesy of G. Liu.

1. Burghard, H.C., and N.S. Stoloff, Cleavage Phenomena and Topographic Feature, in Electron Fractography, C.D. Beachem, Editor. 1968, American Society for Testing and Materials: Philadelphia.

2. Phillips, A., Kerlins, V., and B.V. Whiteson, Electron Fractography Handbook. 1965, Wright-Patterson Air Force Base, Ohio: Air Force Materials Laboratory.

3. Kimura, A. and H. Kimura, Hydrogen embrittlement in high purity iron single crystals. Material Science and Engineering, 1986. 77: p. 75-83.

4. Funding was provided through DOE grant GO15045 